\title{
Prognostic significance of ANLN in lung adenocarcinoma
}

\author{
XIANGYU LONG ${ }^{1}$, WEI ZHOU ${ }^{1}$, YUANXING WANG ${ }^{1}$ and SHIQIANG LIU ${ }^{2}$ \\ ${ }^{1}$ Department of Oncology, West China Guang'an Hospital, Sichuan University, \\ Guang'an, Sichuan 638000; ${ }^{2}$ Department of Cardiothoracic Surgery, Nanchong Central Hospital, \\ The Second Clinical College of North Sichuan Medical College, Nanchong, Sichuan 637900, P.R. China
}

Received November 7, 2017; Accepted April 13, 2018

DOI: $10.3892 / \mathrm{ol} .2018 .8858$

\begin{abstract}
Anillin actin binding protein (ANLN) is a biomarker of cancer progression and is overexpressed in lung adenocarcinoma. The aim of the present study was to investigate the role of ANLN protein and RNA in the development of lung adenocarcinoma. The ANLN protein sequence was downloaded from The National Centre for Biotechnology information, RNA sequencing (RNA-seq) data was obtained from The Cancer Genome Atlas database. All immunohistochemical staining pictures were adapted from the Human Protein Atlas. PyMOL software was employed to predict protein functional changes in response to mutations. Gene Set Enrichments Analysis was employed for pathway analysis. The results indicated that ANLN experiences genetic change and overexpression at the RNA and protein levels in patients with lung adenocarcinoma. Kaplan-Meier survival curve analysis revealed significant differences between high and low RNA-seq expression levels in ANLN, and patients exhibiting higher expression of ANLN had a relatively poor prognosis. Pathway analysis demonstrated that ANLN was involved in developmental processes via the regulation of nuclear division' pathway. In conclusion, ANLN has potential for use as a diagnostic and prognostic biomarker to diagnoseand predict the outcome of lung adenocarcinoma.
\end{abstract}

\section{Introduction}

According to 2015 statistics, lung cancer is considered to be the most common type of cancer and the leading cause of cancer-associated mortality in China (1). Similar statistics have been observed worldwide, with lung cancer being diagnosed in 1.8 million people in 2012, resulting in 1.6 million cases of mortality, rendering it the most common cause of cancer-associated mortality in men and the second most

Correspondence to: Professor Shiqiang Liu, Department of Cardiothoracic Surgery, Nanchong Central Hospital, The Second Clinical College of North Sichuan Medical College, 97 South Renmin Road, Nanchong, Sichuan 637900, P.R. China

E-mail: liushiqiangls1979@163.com

Key words: anillin actin binding protein, lung adenocarcinoma, biomarker, bioinformatics common in women (2). Adenocarcinoma is the most common form of lung cancer, originating in peripheral lung tissue and causing almost $40 \%$ all lung cancer cases (3). The most important risk factor associated with lung adenocarcinoma is tobacco smoking. Obtaining a reliable diagnosis and identifying prognostic markers for lung cancer remains a challenge.

The anillin actin binding protein $(A N L N)$ gene is located on chromosome $7 \mathrm{p} 14.2$, and encodes a protein consisting of 1,124 amino acids that form 4 structural domains, including a myosin- and actin-binding domain, a RhoA-binding domain and a C-terminal pleckstrin homology domain $(4,5)$. The ANLN protein is located in the nucleus, cytoplasm, cytoskeleton, cleavage furrow and cell cortex, and is expressed in adult placenta, testis, and the spinal cord, and in numerous fetal organs (6). ANLN was initially characterized as a human homologue of anillin, a Drosophila actin-binding protein that is present in the cortex following breakdown of the nuclear envelope, and in the cleavage furrow during cytokinesis (7). Anillin serves an important role in cell-cycle progression and in the assembly of the actin and myosin contractile ring that separates daughter cells (6). Importantly, anillin has been demonstrated to be a substrate of the anaphase-promoting complex/cyclosome (APC/C), a typeof ubiquitin ligase that controls mitotic progression (8).

As summarized above, anillin is a conserved protein, which functions in cytoskeletal dynamics during cellularization and cytokinesis. Previous studies have indicated that knockdown of anillinresults in cleavage furrow ingression and failure of cytokinesis in multinucleated monkey BS-C-1 cells (9). The association between cell cycle regulation and carcinoma is well established. ANLN has been indicated to be a marker of poor prognosis, and associated with aggressive cancer phenotypes (10). The role of anillin as a regulator of the cell cycle serves an important role in breast and pancreatic cancer invasion $(11,12)$. ANLN has been developed as a clinically applicable immunohistochemistry-based prognostic biomarker for hepatocellular carcinoma (13). Phylogenetic analysis aims to understand the conservation characterization of analyzed proteins. In the present study, the significance of ANLN protein in human lung adenocarcinoma cancer progression was investigated using The Cancer Genome Atlas (TCGA) database.

\section{Materials and methods}

Data acquisition. The ANLN protein sequences of 11 species (Homo Sapiens, Chimpanzee, Mouse, Zebrafish, Chicken, 
Dog, Sheep, Cattle, Rhesus monkey, Rat and Pig) were downloaded from UniProt (http://www.uniprot.org/) and the National Center for Biotechnology Information (NCBI; https://www.ncbi.nlm.nih.gov/). The mutation statuses of ANLN were checked using cBioPortal for Cancer Genomics (http://www.cbioportal.org). Protein sequence alignment was conducted using Molecular Evolutionary Genetics Analysis software (version 7; MEGA7) (14). The PyMOL Molecular Graphics System (version 1.8; Schrödinger, LLC, New York City, NY, USA) was employed to predict protein functional changes in response to mutations. All immunohistochemical staining images (Fig. 2) were adapted from the Human Protein Atlas (http://www.proteinatlas.org/humancell). ANLN clinical and RNA-seq data were downloaded from The Cancer Genome Atlas (TCGA; https://portal.gdc.cancer.gov/). Gene set enrichment analysis (GSEA, Desktop version 3.0) (15) was used to determine whether any prior-defined pathway or set of genes demonstrated statistically significant differences between two biological states (e.g., phenotypes, RNA-seq expression level in current analysis).

Phylogenetic analysis. Phylogenetic relationships in ANLN expression among the 11 different species were determined using MEGA7 (14). A total of 11 amino acid sequences were analyzed, and all phylogenetic positions containing missing data were eliminated. There were a total of 157 positions in the final dataset. The evolutionary history was inferred using the Neighbor-Joining method (16). The phylogenetic tree was drawn to scale, using branch lengths in the same units as those of the evolutionary distances (number of amino acid substitutions per site), which were calculated using the Poisson correction method (17).

Statistical analysis. All statistical analyses were conducted using R software (Version 3.3.1, http://www.R-project.org/). Monte Carlo simulation (18) was employed to compare RNA-seq expression levels between patients with lung adenocarcinoma and normal control tissues. $\chi^{2}$ was used to analyze differences in survival. The false discovery rate (FDR) method was employed to conceptualize the rate of type I errors in null hypothesis testing when conducting multiple comparisons in GSEA analysis (19).

\section{Results}

ANLN mutations and evolutionary conservation in lung adenocarcinoma. The identified mutations of ANLN in cancer include amplification, deletion and single nucleotide polymorphism (SNP) mutations (missense, nonsense and splice errors) (TCGA database, https://cancergenome.nih.gov/). The mutation rate has been demonstrated to differ according to the type of cancer, ranging from $19.6 \%$ in prostate cancer (20) to $0.2 \%$ in clear cell renal cell carcinoma (21). A total of 27 mutations were identified in 446 patients with lung adenocarcinoma (Fig. 1A and B) using TCGA, including 12 amplifications, 2 deletions and 13 SNP mutations. The 13 SNP mutations included $4 \mathrm{G}>\mathrm{T}, 3 \mathrm{G}>\mathrm{A}, 2 \mathrm{G}>\mathrm{C}, 1 \mathrm{C}>\mathrm{T}$ and $1 \mathrm{C}>\mathrm{A}$ substitutions, among which 5 mutations influenced conserved amino acids sequences (Fig. 1A). A phylogenetic tree was created from the evolutionary analysis performed using MEGA7 software
(Fig. 1C). The optimal phylogenetic tree is presented, with a sum branch length of 0.897 . The ANLN protein sequences from Homo sapiens and Chimpanzee are closely associated, indicating a recent common ancestor. Protein function prediction conducted with PyMOL suggested that these mutations (S594N, G659V, I1014V and R1026L) may influence ionic bonds and $\beta$-folding (Fig. 1D) in the ANLN protein. However, whether these mutations cause gain or loss function remains to be elucidated.

ANLN expression in lung adenocarcinoma. Immunohistochemical staining of the ANLN protein indicated that it is located in the nucleus, and that patients with lung adenocarcinoma exhibit increased ANLN expression compared with healthy controls (Fig. 2). ANLNRNA-seq levels were increased in patients with lung adenocarcinoma compared with normal control tissues (Fig. 3A; Monto Carlo Simulation, $\mathrm{P}<0.001)$ and were significantly associated with high Tumor-Node-Metastasis pathological stage (Fig. 3B; $\mathrm{P}<0.05)$, suggesting that ANLN can predict the disease progression and outcome of patients with lung adenocarcinoma. Kaplan-Meier survival curve analysis demonstrated that the curves for high and low expression of ANLN were significantly different, and that high expression of ANLN was predictive of a poor outcome (Fig. 3C). However, it ANLN RNA-seq levels were not demonstrated to be different between patients with and without ANLN mutations (Fig. 3D).

ANLN specified pathway in lung adenocarcinoma. A total of 59 pairs of lung adenocarcinoma RNA-seq data were extracted for further pathway analysis. A total of 24 Gene Ontology sets and 178 Kyoto Encyclopedia of Genes and Genomes pathways were demonstrated to be associated with ANLN expression (All analysis was done by using GSEA software). The results of GSEA indicated that the pathway of 'regulation of nuclear division' (Fig. 4A and B) was enriched in patients with lung adenocarcinoma (FDR, $\mathrm{q}=0.023$ ). The enrichment score was 0.464 and the normalized enrichment score was 1.834 (Fig. 4C).

\section{Discussion}

The present study indicates that ANLN may be a potential diagnostic and prognostic biomarker for lung adenocarcinoma based on the following findings. A $6 \%$ incidence of genetic change in $A N L N$ was identified in patients with lung adenocarcinoma. Approximately 50\% amplifications (12/27) lead to high expression of ANLN (Fig. 1B), however, the association between other mutations and ANLN protein function require further investigation. Immunohistochemical staining of ANLN in patients with lung adenocarcinoma revealed that it was more highly expressed in adenocarcinoma tissues than in normal tissues, which was also supported by the RNA-seq comparison. The ANLN RNA-seq expression level was associated with TNM stage in lung adenocarcinoma, as well as overall survival rate, suggesting that high expression of $A N L N$ in lung adenocarcinoma is predictive of poor prognosis. GSEA identified that the pathway of 'regulation of nuclear division', involving ANLN, was significantly upregulatedin subjects with lung adenocarcinoma compared with normal controls. 
A

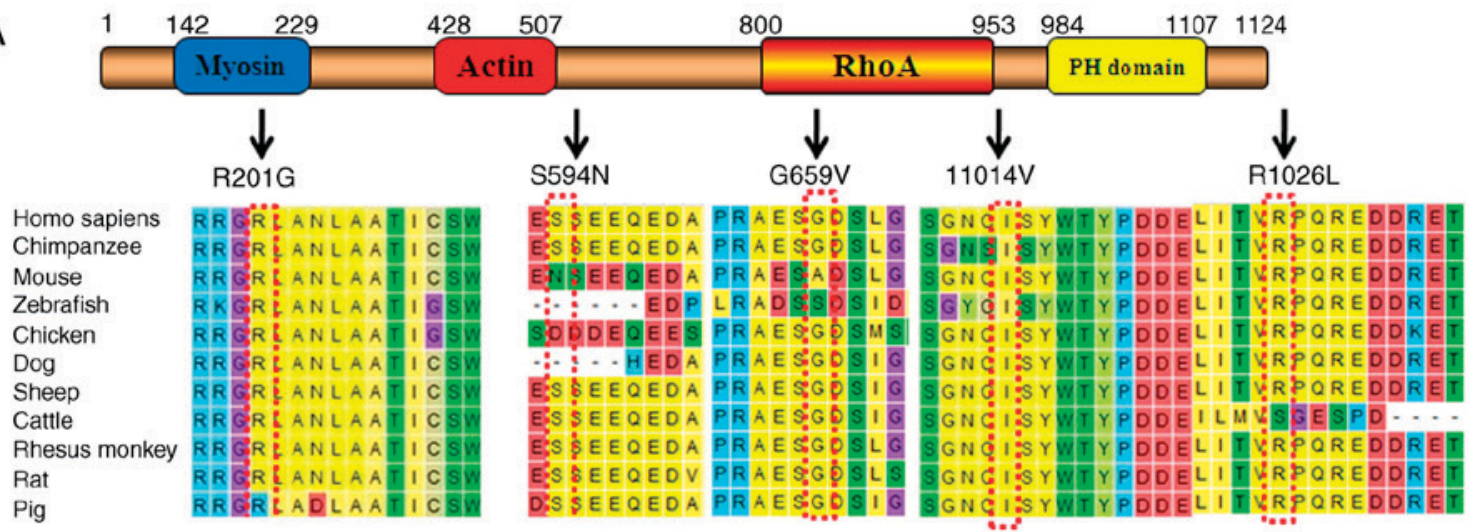

B

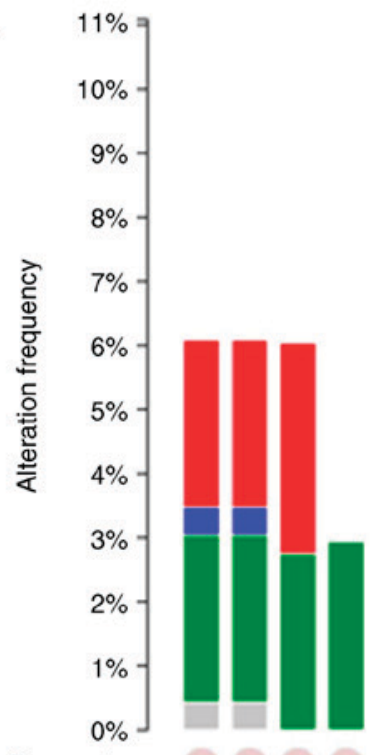

C

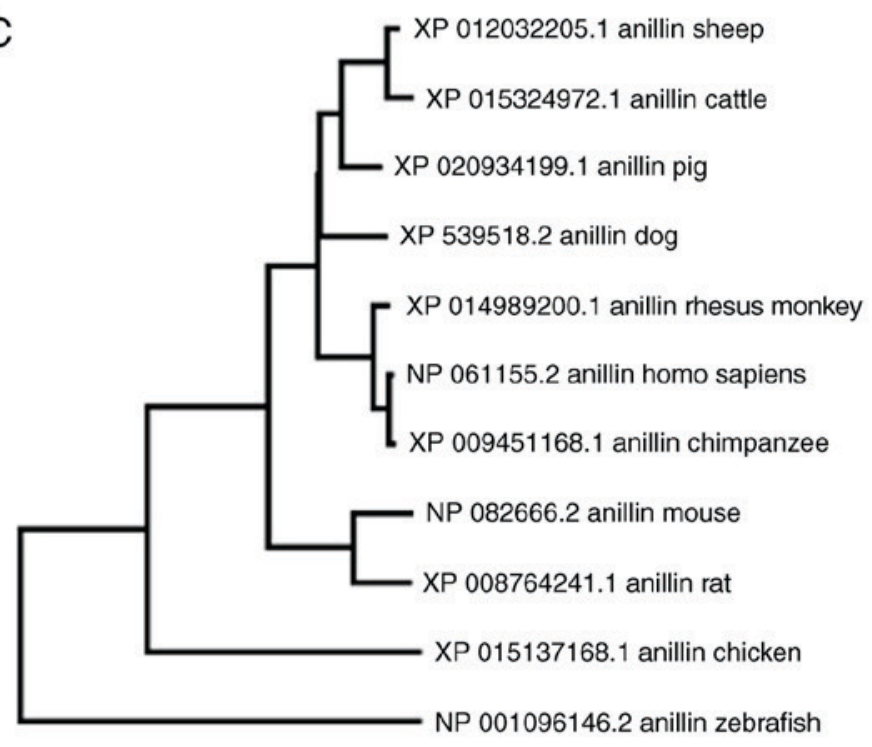

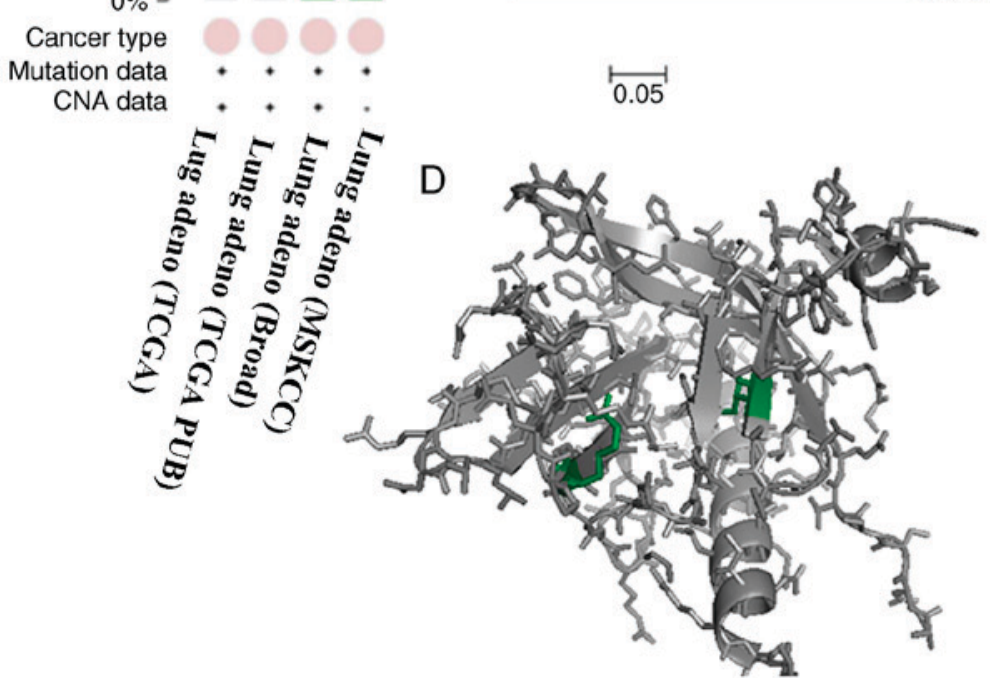

Figure 1. (A) The primary structure of the ANLN protein and how 5 mutations alter the sequence in 11 species using MEGA7.0 software. (B) ANLN mutations in patients with lung adenocarcinoma using cBioPortal (Four lung adenocarcinoma researches from TCGA database, including Lung adenocarcinoma (TCGA), Lung adenocarcinoma (TCGA PUB), Lung adenocarcinoma (Broad) and Lung adenocarcinoma (MSKCC) citied in the TCGA database. Red section is amplification, green is mutation, blue is deep deletion, and grey is multiple alterations). (C) The phylogenetic relationships regarding the ANLN protein among 11 species using MEGA7.0 software. (D) Prediction of changes in the ANLN protein as a result of mutations S594N, G659V, I1014V and R1026L using PyMOL software. Green indicates alternative positions of mutations S594N, G659V, I1014V and R1026L. ANLN, anillin actin binding protein.

Suzuki et al (22) have investigated the significance of $A N L N$ gene in lung cancer using cDNA microarray. In their latest functional experiment, the growth of non-small-cell lung carcinoma (NSCLC) was suppressed using $A N L N$ small interfering RNAs (22). Furthermore, induction of exogenous overexpression of $A N L N$ increased the migratory ability of mammalian cells via interaction with RhoA. Univariate analysis of gene expression in 66 patients with squamous cell carcinoma who had undergone surgical resection identified that $A N L N$ had significant prognostic value (22). This was 

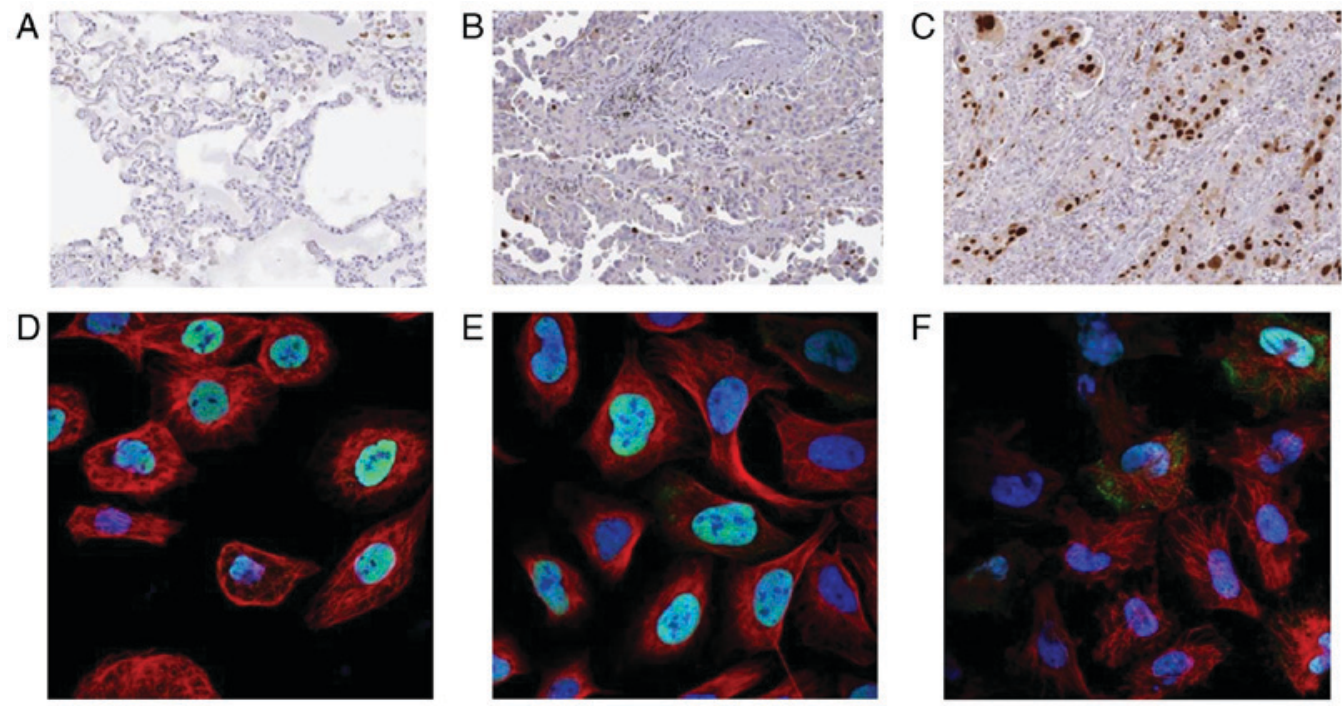

Figure 2. (A) Immunohistochemical staining of ANLN in normal lung tissues x20 magnification, (B) in lung squamous cell carcinoma tissue (x20 magnification), and (C) in adenocarcinoma carcinoma tissue (x20 magnification). ANLN is normally located in the nucleoplasm. Red staining indicates the microtubules, blue staining indicates the nuclei and green staining indicates ANLN expression in (D) A-431 cells (x100 magnification), (E) U-2 OS cells (x100 magnification), and (F) U-251 MG cells (x100 magnification). Brown color indicated ANLN protein staining. All immunohistochemical images were adapted from the Human Protein Atlas (proteinatlas.org/humancell). ANLN, anillin actin binding protein.

A

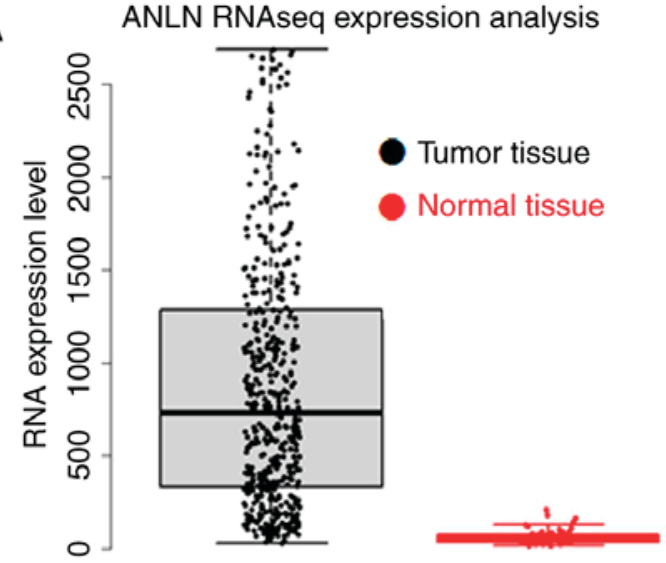

C

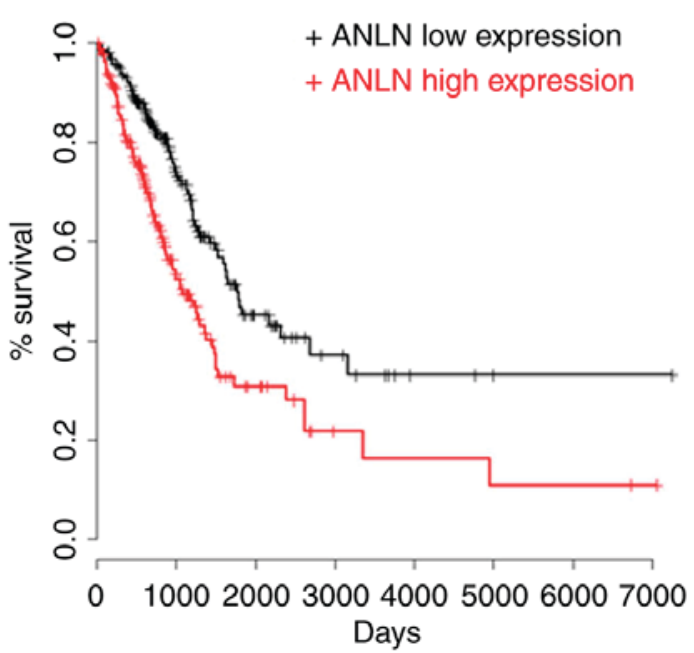

B

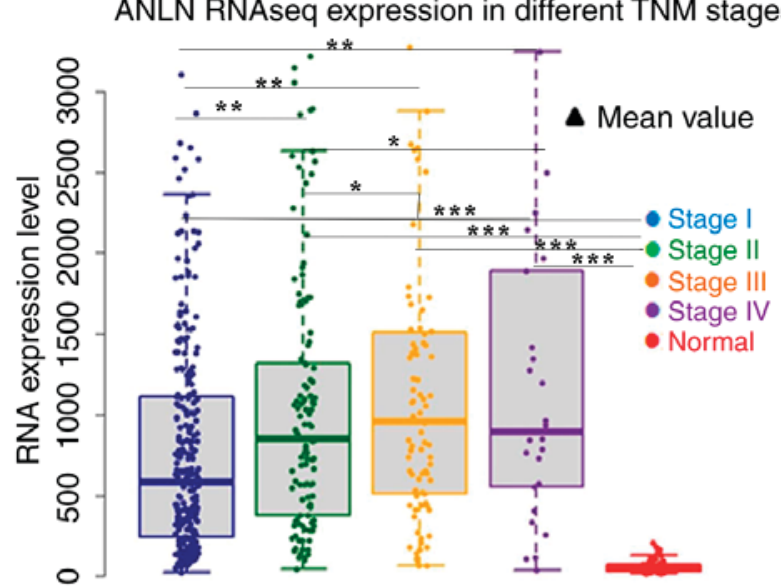

$\mathrm{D}$

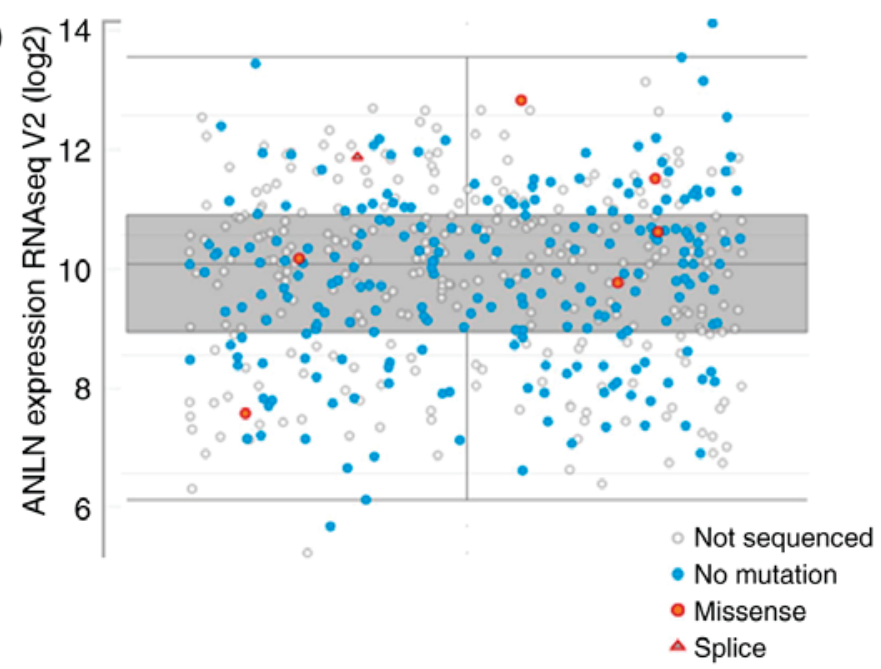

Figure 3. (A) Comparison of ANLN RNA-seq expression between tumor tissues and normal tissues (Monte Carlo Simulation, $\mathrm{P}<0.001$ ). (B) ANLN RNA-seq expression increases with TNM stage (Monte Carlo Simulation, Stage I vs. Stage II, P $<0.01$; Stage I vs. Stage III, P $<0.01 ;$ Stage I vs. Stage IV, P $<0.01 ;$ Stage II vs. Stage III, P<0.05; Stage II vs. Stage IV, P<0.05; Stage III vs. Stage IV, P $>0.05$; All Stage vs. Normal, $\mathrm{P}<0.001$ ). (C) Survival curve analysis of $A N L N$ RNA-seq expression. The two curves are significantly difference ( $\mathrm{P}=0000138)$. (D) ANLN RNA-seq expression in patients with different mutations or without mutation. ANLN, anillin actin binding protein; RNA-seq, RNA sequencing; TNM, Tumor-Node-Metastasis. ${ }^{* * *} \mathrm{P}<0.001 ;{ }^{* * *} \mathrm{P}<0.01 ;{ }^{*} \mathrm{P}<0.05$. 


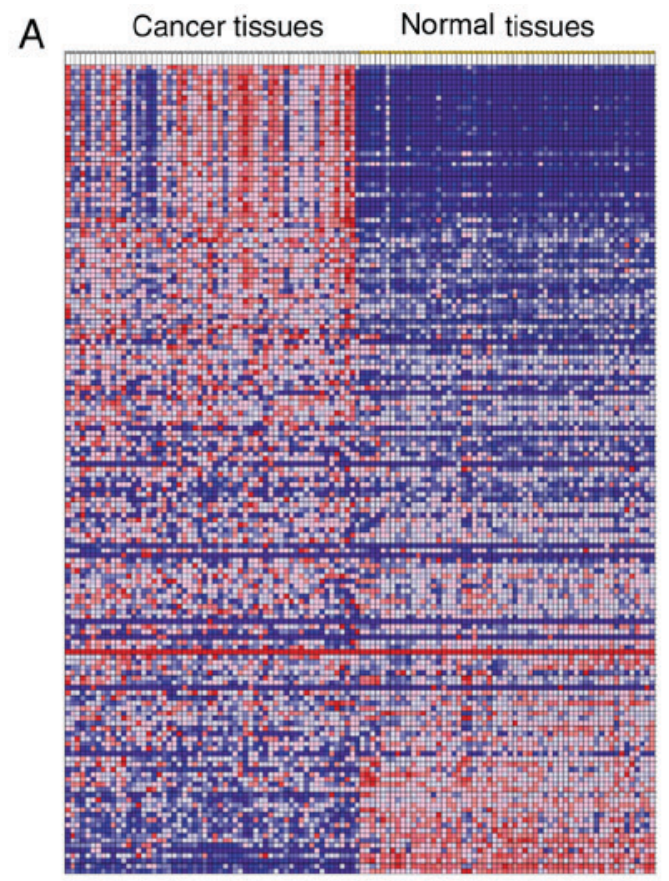

B

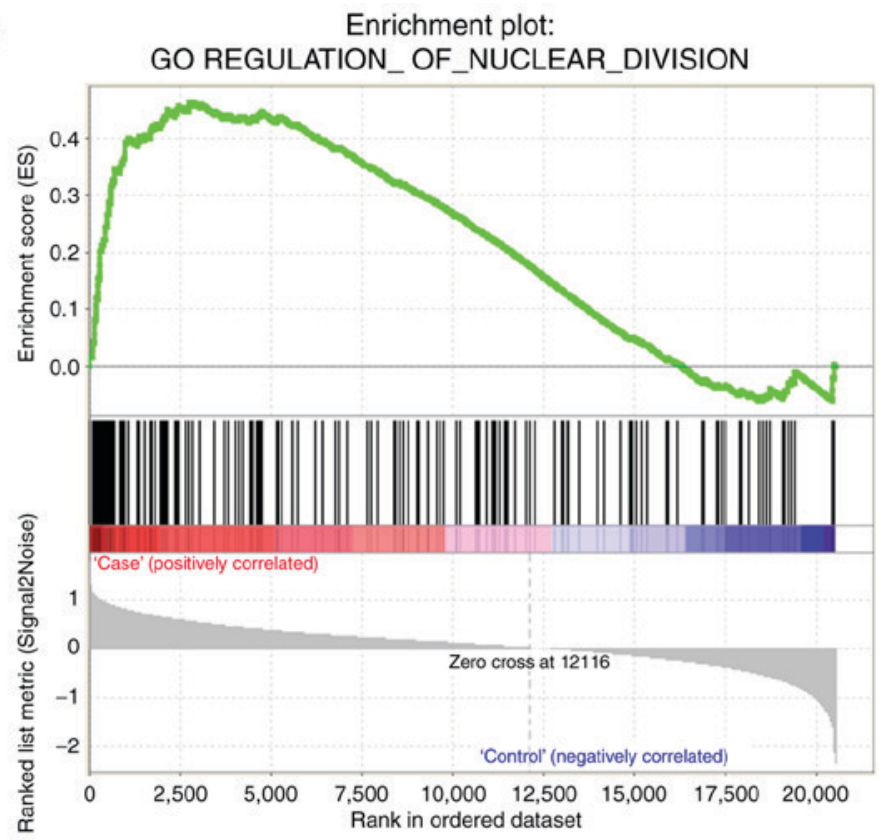

- Enrichment profile - Hits - - Ranking metric scores

C
\begin{tabular}{|l|l|}
\hline Enrichment score (ES) & 0.5273745 \\
\hline Normalized enrichment score (NES) & 1.8370199 \\
\hline Nominal p-value & 0.014 \\
\hline FDR p-value & 0.045 \\
\hline FWER p-value & 0.033 \\
\hline
\end{tabular}

Figure 4. (A) A heat map presenting gene expression in the 'regulation of nuclear division' pathway (Red color indicated high expression, and blue color indicated low expression). (B) GSEA pathway analysis of the 'regulation of nuclear division' pathway. (C) GSEA analysis parameters. GSEA, Gene set enrichment analysis; GO, gene ontology; FDR, false discovery rate; FWER, family-wise error rate.

positively validated in an independent study with a separate cohort of 26 patients (23). All of the above results were supported by the present study.

The regulation of nuclear division pathway, involving ANLN denotes a cell cycle process that comprises the steps by which the nucleus of a eukaryotic cell divides, including the condensation of chromosomal DNA into a highly compact form (24). Inheritance of a defective genome and its mitotic proliferation can lead to pathological conditions, including numerous types of cancer (25). The role of anillin as a cell cycle regulator has been demonstrated to serve an important role in carcinoma invasion (26). However, the detailed mechanisms behind these effects require further investigation.

Limitations of the present study include its retrospective nature, and the use of external databases. The majority of patients on TCGA database were of European descent, and further research using a boarder patient spectrum is required.

In conclusion, the present study established that ANLN protein is a potential prognostic marker for lung adenocarcinoma. However, further investigation is required to elucidate the mechanism of the involvement of ANLN in cell cycle-regulation and the development of adenocarcinoma.

\section{Acknowledgements}

Not applicable.

\section{Funding}

No funding received.

\section{Availability of data and materials}

In the current investigation, the ANLN protein sequences were downloaded from UniProt (uniprot.org/) and also the National Center for Biotechnology Information (NCBI; ncbi.nlm.nih.gov/). ANLN clinical and RNA-seq data were downloaded from The Cancer Genome Atlas (TCGA; portal.gdc.cancer.gov/).

\section{Authors' contributions}

$\mathrm{XL}, \mathrm{WZ}$ and YW performed the sequencing and statistical analyses and manuscript preparing. SL was responsible for study conception and design. 


\section{Ethics approval and consent to participate}

Not applicable.

\section{Consent for publication}

Not applicable.

\section{Competing interests}

The authors declare that they have no competing interests.

\section{References}

1. Chen W, Zheng R, Baade PD, Zhang S, Zeng H, Bray F, Jemal A $\mathrm{Yu}$ XQ and He J: Cancer statistics in China, 2015. CA Cancer J Clin 66: 115-132, 2016

2. World Health Organization (WHO): Global status report on alcohol and health 2014. WHO, Geneva, 2014.

3. Wu-Williams AH, Dai XD, Blot W, Xu ZY, Sun XW, Xiao HP, Stone BJ, Yu SF, Feng YP and Ershow AG: Lung cancer among women in north-east China. Br J Cancer 62: 982-987, 1990.

4. Strausberg RL, Feingold EA, Grouse LH, Derge JG, Klausner RD, Collins FS, Wagner L, Shenmen CM, Schuler GD, Altschul SF, et al: Generation and initial analysis of more than 15,000 full-length human and mouse cDNA sequences. Proc Natl Acad Sci USA 99: 16899-16903, 2002.

5. Ota T, Suzuki Y, Nishikawa T, Otsuki T, Sugiyama T, Irie R, Wakamatsu A, Hayashi K, Sato H, Nagai K, et al: Complete sequencing and characterization of 21,243 full-length human cDNAs. Nat Genet 36: 40-45, 2004.

6. Straight AF, Field CM and Mitchison TJ: Anillin binds nonmuscle myosin II and regulates the contractile ring. Mol Biol Cell 16: 193-201, 2005.

7. Oegema K, Savoian MS, Mitchison TJ and Field CM: Functional analysis of a human homologue of the Drosophila actin binding protein anillin suggests a role in cytokinesis. J Cell Biol 150 $539-552,2000$.

8. Monzo P, Gauthier NC, Keslair F, Loubat A, Field CM Le Marchand-Brustel Y and Cormont M: Clues to CD2-associated protein involvement in cytokinesis. Mol Biol Cell 16: 2891-2902, 2005 .

9. Oegema K, Savoian MS, Mitchison TJ and Field CM: Functional analysis of a human homologue of the Drosophila actin binding protein anillin suggests a role in cytokinesis. J Cell Biol 150: 539-552, 2000.

10. Ronkainen H, Hirvikoski P, Kauppila S and Vaarala MH: Anillin expression is a marker of favourable prognosis in patients with renal cell carcinoma. Oncol Rep 25: 129-133, 2011.
11. Wang Z, Chen J, Zhong MZ, Huang J, Hu YP, Feng DY, Zhou ZJ, Luo X, Liu ZQ, Jiang WZ and Zhou WB: Overexpression of ANLN contributed to poor prognosis of anthracycline-based chemotherapy in breast cancer patients. Cancer Chemother Pharmacol 79: 535-543, 2017.

12. Olakowski M, Tyszkiewicz T, Jarzab M, Krol R, Oczko-Wojciechowska M, Kowalska M, Kowal M, Gala GM, Kajor M, Lange D, et al: NBL1 and anillin (ANLN) genes over-expression in pancreatic carcinoma. Folia Histochem Cytobiol 47: 249-255, 2009.

13. Kim H, Kim K, Yu SJ, Jang ES, Yu J, Cho G, Yoon JH and Kim Y: Development of biomarkers for screening hepatocellular carcinoma using global data mining and multiple reaction monitoring. PLoS One 8: e63468, 2013.

14. Kumar S, Stecher G and Tamura K: MEGA7: Molecular evolutionary genetics analysis version 7.0 for bigger datasets. Mol Biol Evol 33: 1870-1874, 2016.

15. Subramanian A, Kuehn H, Gould J, Tamayo P and Mesirov JP GSEA-P: A desktop application for gene set enrichment analysis. Bioinformatics 23: 3251-3253, 2007.

16. Saitou N and Nei M: The neighbor-joining method: A new method for reconstructing phylogenetic trees. Mol Biol Evol 4: 406-425, 1987.

17. Zuckerkandl E and Pauling L: Evolutionary divergence and convergence in proteins. Evol Genes Proteins 97: 97-166, 1965.

18. Mahadevan S: Monte Carlo Simulation. In: Mechanical Engineering. Marcel Dekker, New York, NY and Basel, pp123-146, 1997.

19. Benjamini Y and Hochberg Y: Controlling the false discovery rate: A practical and powerful approach to multiple testing. J R Statistical Soc Ser B (Methodological) 57: 289-300, 1995.

20. Beltran H, Prandi D, Mosquera JM, Benelli M, Puca L, Cyrta J, Marotz C, Giannopoulou E, Chakravarthi BV, Varambally S, et al: Divergent clonal evolution of castration-resistant neuroendocrine prostate cancer. Nat Med 22: 298-305, 2016.

21. Cancer Genome Atlas Research Network. Comprehensive molecular characterization of clear cell renal cell carcinoma. Nature 499: 43-49, 2013.

22. Suzuki C, Daigo Y, Ishikawa N, Kato T, Hayama S, Ito T, Tsuchiya E and Nakamura Y: ANLN plays a critical role in human lung carcinogenesis through the activation of RHOA and by involvement in the phosphoinositide 3-kinase/AKT pathway. Cancer Res 65: 11314-11325, 2005.

23. Skrzypski M, Jassem E, Taron M, Sanchez JJ, Mendez P, Rzyman W, Gulida G, Raz D, Jablons D, Provencio M, et al: Three-gene expression signature predicts survival in early-stage squamous cell carcinoma of the lung. Clin Cancer Res 14: 4794-4799, 2008.

24. Nigg EA: Mitotic kinases as regulators of cell division and its checkpoints. Nat Rev Mol Cell Biol 2: 21-32, 2001.

25. Brinkley BR: Managing the centrosome numbers game: From chaos to stability in cancer cell division. Trends Cell Biol 11: $18-21,2001$.

26. Park MT and Lee SJ: Cell cycle and cancer. J Biochem Mol Biol 36: 60-65, 2003. 